\title{
Perfil clínico de pacientes submetidos à cirurgia de revascularização do miocárdio e troca valvar em um hospital terciário da região Sul do Brasil
}

\section{Clinical profile of patients submitted to coronary artery bypass graft surgery and valve replacement in a tertiary hospital of Southern Brazil}

\author{
Carlos Cassiano Figueiró da Silva, Mariana Brondani de Mello, Amanda Albiero Real, \\ Isabella Martins de Albuquerque.
}

Como citar este artigo:

SILVA, C. C. F.; MELLO, M. B.; REAL, A. A.; ALBUQUERQUE, I.M. Perfil clínico de pacientes submetidos à cirurgia de revascularização do miocárdio e troca valvar em um hospital terciário da região Sul do Brasil. Revista Saúde (Sta. Maria). 2019; 45 (2)

\section{Autor correspondente:} Nome: Isabella Martins de Albuquerque

E-mail: albuisa@gmail.com Telefone: (55) 99643-9577 Formação Profissional:

Formada em Fisioterapia pela Universidade Federal de Santa Maria (UFSM), e Doutora em Ciências Médicas pela Universidade Federal do Rio Grande do Sul (UFRCS).

Filiação Institucional: Universidade Federal de Santa Maria (UFSM) Endereço para correspondência: Avenida Roraima n: 1000 Bairro: Camobi

Cidade: Santa Maria Estado: Rio Grande do Sul CEP: 97 105-900

Data de Submissão:

01/07/2019

Data de aceite:

04/08/2019

Conflito de Interesse: Não há conflito de interesse

\section{(cc) $\mathrm{B} Y-\mathrm{Ne}-\mathrm{ND}$}

\section{RESUMO}

Objetivo: Analisar o perfil clínico de pacientes submetidos à cirurgia de revascularização do miocárdio (CRM) e troca valvar (TV) em um hospital terciário na região Sul do Brasil. Métodos: Estudo transversal e retrospectivo com coleta de dados obtidos do registro de prontuário de pacientes submetidos à cirurgia cardíaca no período de abril de 2016 a março de 2017 no Hospital Universitário de Santa Maria (HUSM). A normalidade das variáveis foi avaliada pelo teste de Shapiro-Wilk. Os dados de prevalência foram apresentados em frequências absolutas e porcentagens. Para verificar a correlação entre os fatores de risco e o tempo de internação na unidade de cardiologia intensiva (UCI) assim como entre os fatores de risco e as complicações no pós-operatório (PO), foi utilizado o coeficiente de correlação de Pearson e para efeito de significância estatística foi considerado um $p<0,05$. Resultados: A amostra composta por 44 pacientes, com média de idade de $62,31 \pm 10,85$ anos, sendo 28 (64\%) do sexo masculino. 0 procedimento mais frequente foi a CRM em 28 pacientes (64\%), seguida de TV em 9 (20\%) e concomitante CRM e TV em 7 (16\%). Os fatores de risco mais prevalentes foram diabetes mellitus (DM) (82\%), hipertensão arterial sistêmica (HAS) (82\%) e história pregressa de tabagismo (36\%). O tempo de circulação extracorpórea (CEC) em média foi de 102,88 \$38,11 minutos e tempo total de cirurgia em média de $276,7 \pm 82,86$ minutos. Não houve correlação significativa entre os fatores de risco e o tempo de internação na $U C l(r=0,125 ; p=0,416)$ e entre os fatores de risco e as complicações no PO ( $r=0,041 ; p=0,791)$. Conclusão: Os pacientes deste estudo em sua maioria foram do sexo masculino e constatou-se importante prevalência de comorbidades como HAS e DM. Tais achados podem revelar uma condição de maior gravidade corroborando para o desenvolvimento de doenças cardiovasculares.

PALAVRAS-CHAVE: Cirurgia Torácica; Procedimentos Cirúrgicos Cardíacos; Fatores de Risco; Complicações Pós-Operatórias.

\section{ABSTRACT}

Objective: To analyze the clinical profile of the patients submitted to coronary artery bypass graft $(\mathrm{CABG})$ and valve replacement (VT) at a tertiary hospital in the southern region of Brazil. Methods: Cross-sectional and retrospective study with data collection obtained from the registry of patients submitted to cardiac surgery from April 2016 to March 2017 in the Hospital Universitário de Santa Maria (HUSM). The normality of the variables was evaluated by the Shapiro-Wilk test. Prevalence data were presented in absolute frequencies and percentages. In order to verify the correlation between risk factors and length of hospital stay in the intensive cardiology unit (ICU) as well as between risk factors and postoperative complications (PO), the Pearson correlation coefficient was used and the significance level was set at $p<0.05$ for all analyses. Results: The sample consisted of 44 patients, with average age of $62.31 \pm 10.85$ years, of which $28(64 \%)$ were male. The most frequent procedure was 
CABG in 28 patients (64\%), followed by valve replacement in $9(20 \%)$ and concomitant CABG and valve replacement in 7 (16\%). The most prevalent risk factors observed in these patients were diabetes mellitus (DM) (82\%), systemic arterial hypertension (SAH) (82\%) and previous history of smoking (36\%). The average extracorporeal circulation time (ECC) was $102.88 \pm 38.11$ minutes and total surgery time was 276.7 \pm 82.86 minutes. There was no significant correlation between risk factors and length of hospital stay in the ICU $(r=0,125, p=0,416)$ and between risk factors and complications in the $\mathrm{PO}(r=0,041, p=0,791)$.Conclusion: The majority of the patients in this study were male, and a significant prevalence of comorbidities such as SAH and DM was observed. These findings may reveal a more serious condition to increase the risk of cardiovascular diseases.

KEYWORDS: Thoracic Surgery; Cardiac Surgical Procedures; Risk Factors; Postoperative Complications.

\section{INTRODUÇÃO}

As doenças cardiovasculares (DCVs) configuram-se como a principal causa de morte no mundo, representando um importante problema clínico e de saúde pública¹. A Organização Mundial da Saúde (OMS) estima que 17,7 milhões de pessoas morreram por DCVs em 2012, representando 31\% de todas as mortes em nível global. Desses óbitos, estimase que 7,4 milhões ocorrem devido à doença arterial coronariana $(\mathrm{DAC})^{2}$, a qual se caracteriza pelo estreitamento das artérias coronárias devido à formação e acúmulo de placas de ateroma+. Além da DAC, a doença valvar constitui uma parcela expressiva de internações por DCVs e sua principal etiologia é a febre reumática que representa $70 \%$ dos casos no Brasil| .

Nesse contexto, os fatores de risco das DCVs têm relação direta com a progressão, evolução e complicações da doença. Dentre eles encontram-se os fatores de risco modificáveis, em que os sujeitos tendem a alterar com a mudança de hábitos de vida e os não modificáveis, os quais têm relação com a herança genética ${ }^{3,5}$. Diversos são os fatores de risco responsáveis pela origem, desenvolvimento e complicações das DCVs tem-se como destaque: idade avançada, hereditariedade, hipertensão arterial sistêmica (HAS), diabetes mellitus (DM), dislipidemia (DLP), sedentarismo, obesidade e tabagismo 5 .

As DCVs podem ter tratamento conservador (clínico/medicamentoso) ou cirúrgico ${ }^{6}$. A cirurgia cardíaca é considerada de grande porte e resulta em importantes repercussões orgânicas alterando os mecanismos fisiológicos dos pacientes. Além disso, conduz a um estado crítico no pós-operatório (PO), culminando em cuidados intensivos a fim de se estabelecer uma boa recuperação dos mesmos ${ }^{7,8}$. Esse procedimento pode ser realizado de maneira reconstrutora, substitutiva ou corretora, o mais utilizado é o de reconstrução, especialmente revascularização miocárdica ${ }^{9}$, que juntamente com as trocas valvares, são intervenções complexas que necessitam de um tratamento adequado em todas as fases operatórias ${ }^{10}$.

Em 2012, foram realizadas mais de 102 mil cirurgias cardíacas no Brasil, destacando-se a cirurgia de revascularização do miocárdio, a qual é o procedimento cirúrgico mais frequentemente realizado pelo Sistema Único de 
Saúde (SUS) $)^{10}$. O avanço da medicina e o aumento da expectativa de vida possibilitaram a esses pacientes um acesso amplo e indicação ao procedimento cirúrgico ${ }^{11}$.

Tendo em vista o caráter complexo das cirurgias cardíacas, esse estudo teve como objetivo analisar o perfil clínico de pacientes submetidos à CRM e troca valvar (TV) em um hospital terciário da região Sul do Brasil.

\section{MÉTODO}

Trata-se de um estudo de caráter transversal e retrospectivo cuja coleta de dados foi realizada em prontuários médicos de pacientes submetidos à CRM e/ou TV no Hospital Universitário de Santa Maria (HUSM) da Universidade Federal de Santa Maria (UFSM), em Santa Maria, Rio Grande do Sul, Brasil, entre abril de 2016 a março de 2017. 0 estudo foi aprovado pelo Comitê de Ética em Pesquisa local sob CAAE n 53561416.9.0000.5346.

Foram incluídos pacientes de ambos os sexos, maiores de 18 anos, sendo excluídos aqueles submetidos aos procedimentos de correção de aneurisma da aorta, angioplastia, valvuloplastia, retirada de mixoma, dissecção da aorta ou atrioseptoplastia e indivíduos cujos prontuários não apresentavam informações suficientes.

A coleta de dados ocorreu com auxílio de um instrumento com questões estruturadas, contendo dados demográficos (sexo, idade, peso, altura), e clínicos (fatores de risco para DCVs, sintomas pré-operatório, informações do procedimento cirúrgico realizado como tempo de CEC e tempo de cirurgia, tempo de internação na UCI e na enfermaria e as complicações apresentadas no PO).

A análise estatística foi realizada utilizando-se o software GraphPadPrism 5 (GraphPad Software Inc., San Diego, CA, EUA). A normalidade das variáveis foi avaliada pelo teste de Shapiro-Wilk. Os dados de prevalência foram apresentados em frequências absolutas e porcentagens. Para verificar a correlação entre os fatores de risco e o tempo de internação na UCl e as complicações no PO foi utilizado o coeficiente de correlação de Pearson e para efeito de significância estatística foi considerado um $p<0,05$.

\section{RESULTADOS}

Foram analisados os prontuários de 97 pacientes de ambos os sexos, com idade entre 29 e 81 anos submetidos à CRM e/ou TV. Foram excluídos 39 prontuários, pois não preencheram os critérios de inclusão, 11 por não apresentarem informações suficientes e 3 por irem a óbito (Figura 1). 


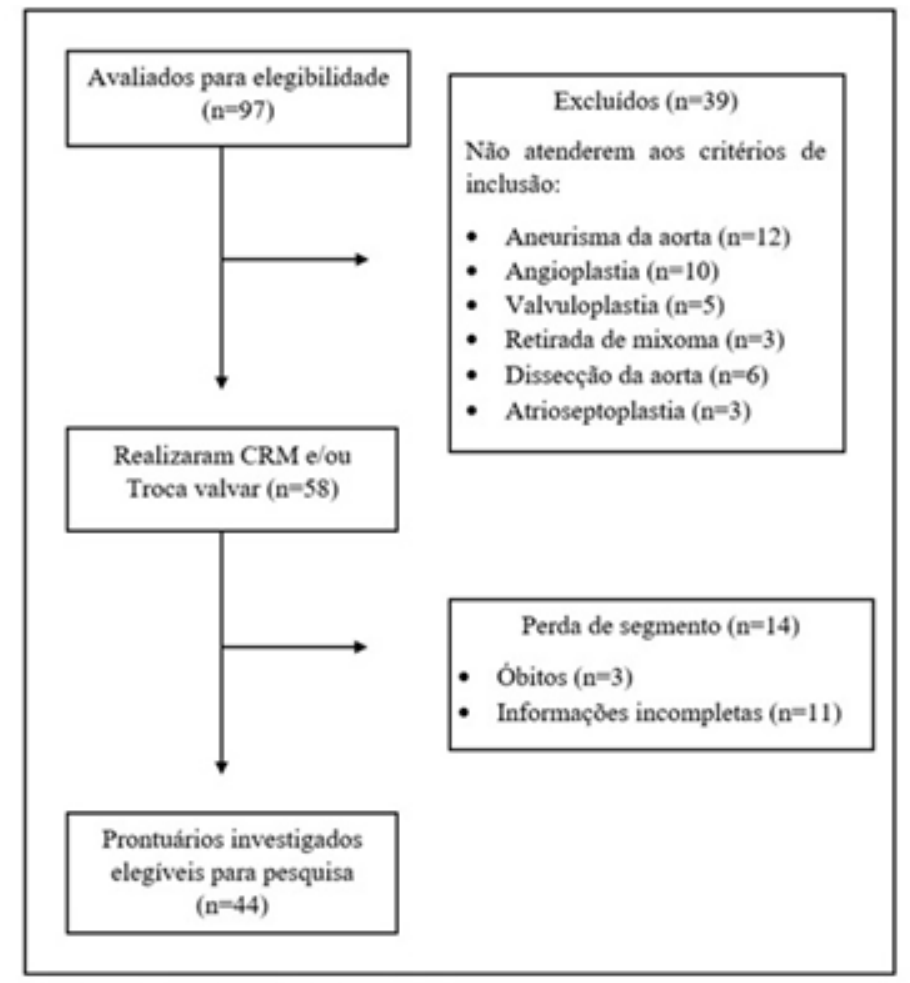

Figura 1 - Fluxograma de composição da amostra

A amostra foi composta por 44 pacientes, com média de idade de 62,31 110,85 anos. A Tabela 1 apresenta as características gerais dos pacientes.

Tabela 1. Caracterização da amostra

\begin{tabular}{|c|c|c|}
\hline Características & $\mathrm{n}$ & $\%$ \\
\hline \multicolumn{3}{|l|}{ Gênero } \\
\hline Masculino & 28 & 64 \\
\hline Feminino & 16 & 36 \\
\hline IAM prévio & 22 & 50 \\
\hline Histórico familiar de cardiopatia & 14 & 32 \\
\hline \multicolumn{3}{|l|}{ Tipo de cirurgia } \\
\hline CRM & 28 & 64 \\
\hline Troca valvar & 09 & 20 \\
\hline CRM e troca valvar & 07 & 16 \\
\hline \multicolumn{3}{|l|}{ Classificação IMC } \\
\hline Normal & 17 & 39 \\
\hline Sobrepeso & 15 & 34 \\
\hline Obesidade grau I & 09 & 20 \\
\hline Obesidade grau II & 03 & 07 \\
\hline \multicolumn{3}{|l|}{ Medicação } \\
\hline AAS & 40 & 91 \\
\hline Betabloqueadores & 35 & 80 \\
\hline \multicolumn{3}{|l|}{ Estatina } \\
\hline IECA & 28 & 64 \\
\hline
\end{tabular}




$\begin{array}{lcc}\text { Diurético } & 17 & 39 \\ \text { Anticoagulante } & 14 & 32 \\ \text { Monitrato de Isossorbida } & 13 & 30 \\ \text { Bloqueadores dos Canais de Ca } & 12 & 27 \\ \text { BRA's } & 07 & 16\end{array}$

Valores em n (\%). IAM: Infarto Agudo do Miocárdio; CRM: Cirurgia de Revascularização do Miocárdio; IMC: Índice de Massa Corporal; AAS: ácido acetilsalicílico; IECA: inibidores da enzima conversora de angiotensina; BRA's: Bloqueadores de receptores da angiotensina.

Os fatores de risco de maior prevalênciasão apresentados na Figura 2.

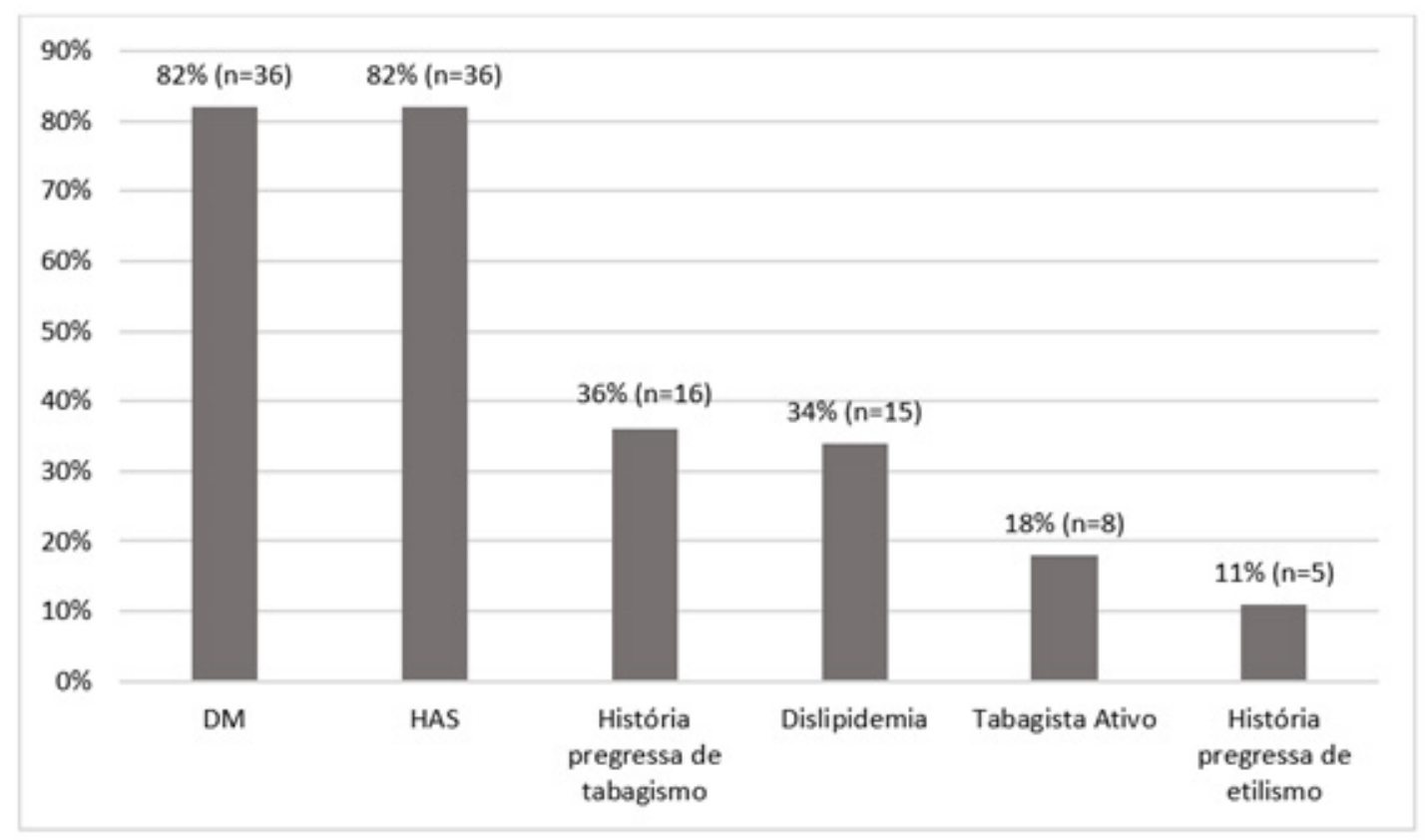

Figura 2. Prevalência de fatores de risco para doença cardiovascular

Em relação aos sintomas pré-operatórios, 59\% (n=26) dos pacientes apresentaram dor torácica retroesternal e precordial, 50\% (n=22) dispneia, 16\% (n=7) dor retroesternal com irradiação para o membro superior esquerdo, 7\% $(n=3)$ apresentaram dor toracolombar e náuseas e apenas $5 \%(n=2)$ referiram dor abdominal. As variáveis clínicas e cirúrgicas são apresentadas na Tabela 2.

Tabela 2. Variáveis cirúrgicas e clínicas da amostra

\begin{tabular}{lc}
\hline Variáveis & Média $\pm \mathrm{DP}$ \\
\hline Tempo de CEC & $102,88 \pm 38,11$ minutos \\
Tempo de cirurgia & $276,70 \pm 82,86$ minutos \\
Tempo de internação na UCl & $5,13 \pm 2,23$ dias \\
Tempo de internação PO & $9,50 \pm 4,53$ dias \\
\hline
\end{tabular}

Valores em média DP. CEC: Circulação extracorpórea; UCl: Unidade de Cardiologia Intensiva; PO: Pós-Operatório; DP: desvio padrão. 
Na Figura 3 são apresentadas as complicações mais prevalentes no PO.

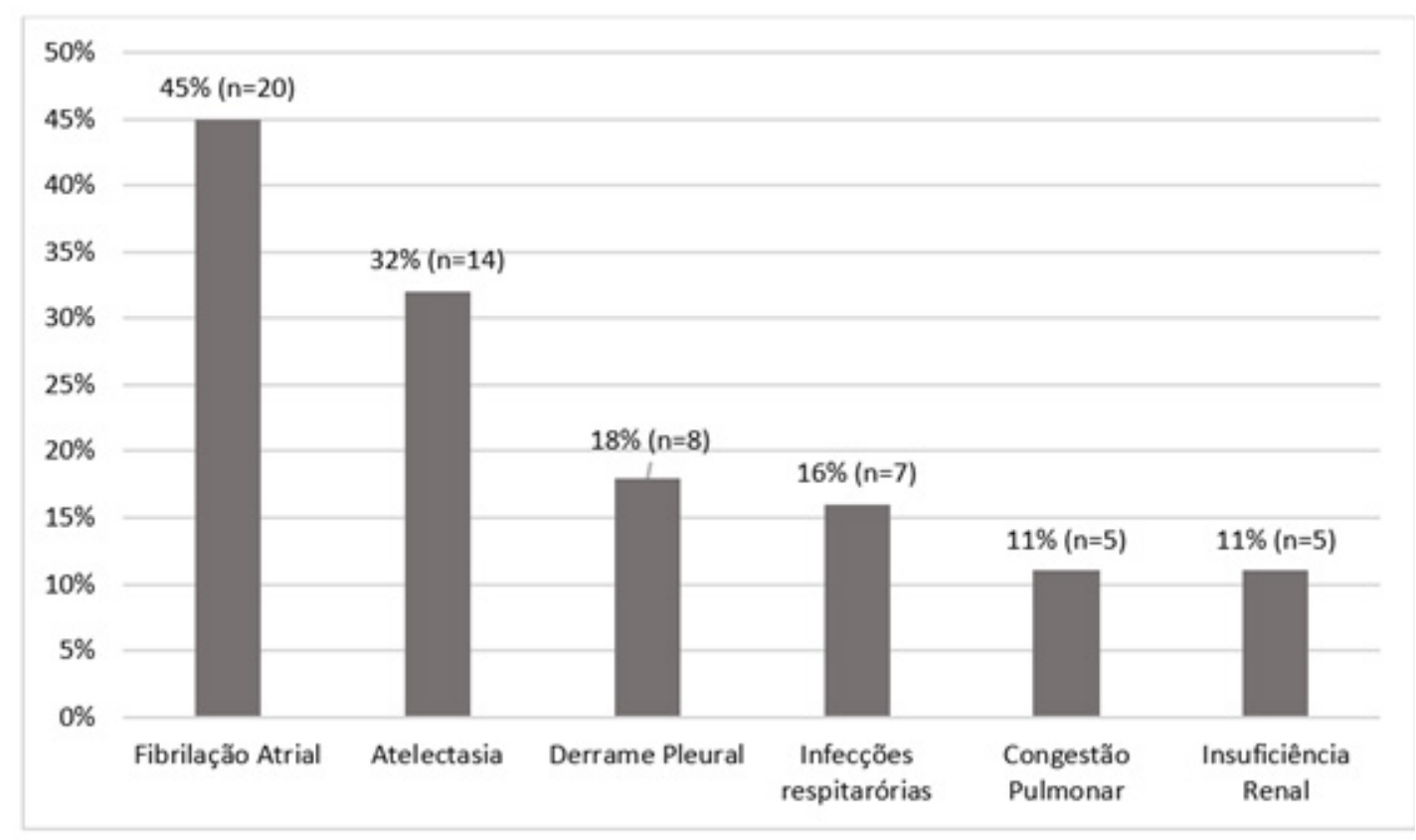

Figura 3. Prevalência de complicações no pós-operatório de cirurgia cardiaca

Não houve correlação significativa entre fatores de risco com tempo de internação na $U C l(r=0,125 ; p=0,416)$, assim como entre os fatores de risco e complicações presentes no PO ( $r=0,041 ; p=0,791)$.

\section{DISCUSSÃO}

Esse estudo buscou caracterizar o perfil e analisar as características clínicas de pacientes submetidos à CRM e/ ou troca valvar, em um hospital terciário na região Sul do Brasil. Houve predomínio de sujeitos do sexo masculino (64\%) e a média de idade foi de 62,31 110,85 anos, embora $68 \%$ não apresentassem histórico familiar de cardiopatia, metade deles apresentaram IAM prévio. A predominância de CRM em relação a outras cirurgias cardíacas, como troca valvar, foi de $62 \%$. Tais achados vão ao encontro dos estudos de Silveira et al. ${ }^{12}$, Araújo et al. ${ }^{13}$ e Cani et al. ${ }^{14}$ no qual houve predomínio do sexo masculino e idade acima de 60 anos, além da CRM ser o procedimento cardíaco mais realizado. Dentro deste contexto, o estudo de Cani et al. ${ }^{14}$ confirma que pacientes que apresentaram IAM prévio e histórico familiar de cardiopatia possuíam fatores de risco modificáveis para DCVs.

Com relação à classificação do IMC, o presente estudo observou que 34\% da amostra apresentava sobrepeso e 39\% peso normal. Tais achados respaldam o estudo de Costa et al. ${ }^{15}$ onde a maioria dos sujeitos submetidos à CRM e/ou troca valvar apresentava-se com sobrepeso $(47,5 \%)$ e $32 \%$ com peso normal. Entretanto, no estudo de Dessote et al. ${ }^{16}$, sujeitos submetidos a esses procedimentos em sua maioria eram portadores de sobrepeso e obesos, visto que essa 
população tem aumentado continuamente entre adultos de ambos os sexos no Brasil e está associada ao surgimento de fatores de risco para DCVs como HAS, DM e DLP17.

Considerando os fatores de risco mais prevalentes para DCVs, no presente estudo houve predominância de DM e HAS. Outros estudos que envolveram o mesmo perfil de pacientes também demostraram que grande parte da população estudada apresentava esses mesmos fatores ${ }^{15,18}$. Entretanto, há uma divergência em relação aos demais fatores de risco, visto que Kaufman et al. ${ }^{19}$ demonstraram em seu estudo que a DLP, a história familiar de DAC e a história pregressa de tabagismo são mais prevalentes que DM. Contudo, o estudo de Caldeira, Soares ${ }^{18}$ demostrou que a DLP e história pregressa de tabagismo são os fatores de risco com maior prevalência após HAS e DM. Além dos já citados, a história pregressa de etilismo também esteve presente em alguns pacientes da amostra, pois estudos vêm demonstrando que esse hábito influencia o surgimento de DCVs ${ }^{12,13}$.

Com relação ao tempo de CEC e ao tempo total de cirurgia, nossos achados demonstraram valores elevados quando comparados a outros estudos envolvendo o mesmo perfil de cirurgias ${ }^{8,20}$. No estudo de Cordeiro et al. ${ }^{21}$, o tempo elevado de CEC foi um dos principais fatores que prolongaram o desmame da ventilação mecânica no pós-operatório de cirurgia cardíaca.

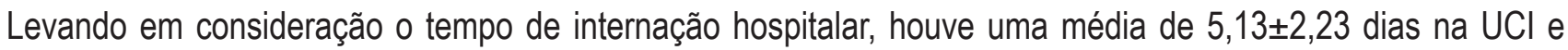
9,5 \pm 4,53 dias na enfermaria hospitalar. Entretanto, outros estudos apresentaram um tempo médio inferior, tal achado possivelmente possa ser explicado devido às rotinas de alta de cada unidade, visto que pacientes que realizam cirurgia cardíaca e não apresentam nenhuma complicação, recebem alta da UCl usualmente no $2^{\circ}$ dia de $\mathrm{PO}^{12,20}$. 0 menor tempo de internação hospitalar está relacionado ao melhor prognóstico, pois o retorno precoce às atividades diárias, bem como o convívio familiar, acelera a reabilitação do paciente submetido à cirurgia cardíaca²2 .

Entre as complicações mais prevalentes encontradas no PO de CRM e TV, destaca-se a fibrilação atrial (FA), presente em $45 \%$ dos pacientes. Tal complicação tem associação com taxas elevadas de mortalidade a curto e longo prazo e com maior prevalência para complicações no $\mathrm{PO}^{23}$. Estudo de Hawkis et al. ${ }^{24}$ demonstrou que a ocorrência de FA no PO foi decorrente de um possível agravamento da insuficiência cardíaca, levando ao aumento da insuficiência renal, assim como oprolongamento do tempo de internação na UTI e na enfermaria. Outro estudo observou que o tempo de internação foi maior nos pacientes que desenvolveram FA após cirurgia cardíaca ${ }^{25}$.

Ainda no contexto de complicações no PO, as pulmonares são eventos frequentes em pacientes que foram submetidos à cirurgia cardíaca ${ }^{26}$. A intensidade da manipulação cirúrgica, grau de sedação, número de drenos pleurais, dor e o uso de CEC são os principais fatores intraoperatórios que alteram a mecânica respiratória no PO imediato ${ }^{27}$.

No presente estudo, a atelectasia foi a complicação pulmonar que ocorreu com maior frequência, o que corrobora com os achados de Oliveira et al. ${ }^{28}$. Na CEC, a falta de perfusão durante o procedimento ocasiona a redução 
do surfactante e o posterior colapso alveolar, o que contribuiu para o desenvolvimento de atelectasias. No recente estudo conduzido por Brown et al..$^{29}$, os autores demonstraram que o derrame pleural também foi uma das complicações pulmonares mais frequentes no PO de cirurgia cardíaca.

Pacientes que apresentam um número maior de complicações no PO tendem a permanecer um tempo significativamente maior em internação hospitalar e na UTT7,14. Embora a população incluída no estudo tenha apresentado diversos fatores de risco para DCVs, complicações no PO e um tempo elevado de internação, não houve correlação significativa entre os fatores de risco e o tempo de internação na UCl assim como entre os fatores de risco e as complicações no PO. Hipotetiza-se que tal achado pode ter ocorrido em função do tamanho amostral do estudo.

Esse estudo apresentou algumas limitações que devem ser consideradas, tais como: o risco de viés de seleção (estudo retrospectivo) e de aferição (dados provenientes de prontuários médicos), assim como ter sido realizado em um único centro. Apesar dessas limitações, o presente estudo constatou que a maioria dos pacientes apresentou um ou mais fatores de risco para o desenvolvimento de DCV, fato esse, que respalda a importância da prevenção através de ações educativas, além da avaliação e intervenção fisioterapêutica adequada no pré-operatório com o objetivo de reduzir possíveis complicações PO e, consequentemente, uma possível redução do tempo de internação hospitalar e na UCl.

\section{CONSIDERAÇÕES FINAIS}

Os resultados do presente estudo demonstraram que a amostra foi composta em sua grande maioria pelo sexo masculino e constatou-se uma importante prevalência de comorbidades como HAS, DM e história pregressa de tabagismo, o que pode revelar uma condição de maior gravidade corroborando para o desenvolvimento de DCVs. Em virtude do caráter complexo da cirurgia cardíaca, nossos achados mostraram características importantes de um grupo significativo de pacientes, assim como sinaliza à atenção que deve ser atribuída às atividades de prevenção da saúde, visto o elevado risco cardiovascular da população.

\section{REFERÊNCIAS}

1. Ribeiro KRA. Pós-operatório de revascularização do miocárdio: complicações e implicações para enfermaาgem. Revista de Pesquisa: Cuidado é Fundamental Online. 2018; 10:254-259.

2. World Health Organization (WHO). Cardiovascular diseases (CVDS). 2017. 
3. Montenegro Sá F, Ruivo C, Graça Santos L, Antunes A, Soares FC, Baptista J, et al. Progressão ultrarrápida de doença coronária ou placa instável não detetada? Rev Port Cardiol. 2018;37:259-264.

4. Tarasoutchi F, Montera MW, Ramos AIO, Sampaio RO, Rosa VEE, Accorsi TAD, et al. Atualização das Diretrizes Brasileiras de Valvopatias: Abordagem das Lesões Anatomicamente Importantes. Arquivos Brasileiros de Cardiologia 2017;109(6Supl.2):1-34.

5. Ribeiro S, Furtado C, Pereira J. Associação entre as doenças cardiovasculares e o nível socioeconómico em Portugal. Revista Portuguesa de Cardiologia. 2013;32(11):847-854.

6. Santos CA, Oliveira MAB, Brandi AC, Botelho PHH, Brandi JCM, Santos MA, et al. Risk factors for mortality of patients undergoing coronary artery bypass graft surgery. Revista Brasileira de Cirurgia Cardiovascular. 2014;28(4):513-520.

7. Koerich C, Lanzoni GMM, Erdmann AL. Factors associated with mortality in patients undergoing coronary artery by-pass grafting. Revista Latino-Americana de Enfermagem. 2016; 24:27-48.

8. Cordeiro AL, GuimarãesAR, Pontes SS, Jesus L, Lima C, Coutinho V. Características clínicas e cirúrgicas de idosos submetidos à cirurgia cardíaca. Revista Pesquisa em Fisioterapia. 2017;7(1):30-35.

9. Barretta JC, Auda JM, Barancelli MDC, Antoniolli D. Pós-operatório em cirurgia cardíaca: refletindo sobre o cuidado de enfermagem. Revista de Pesquisa: Cuidado é Fundamental Online. 2017;9(1):259-264.

10. Dordetto PR, Pinto GC, Rosa TCSC. Pacientes submetidos à cirurgia cardíaca: caracterização sociodemográfica, perfil clínico-epidemiológico e complicações. Revista da Faculdade de Ciências Médicas de Sorocaba. 2016;18(3):144-149.

11. Cani KC, Araujo CLP, Karloh M, Alexandrino DFH, Palú M, Rojas DB, et al. Características clínicas de pacientes submetidos à cirurgia de revascularização do miocárdio. ASSOBRAFIR Ciência, São Paulo. 2015;6(3):43-54.

12. Silveira CR, Santos MBK, Moraes MAP, Souza EN. Desfechos clínicos de pacientes submetidos à cirur- 
gia cardíaca em um hospital do noroeste do Rio Grande do Sul. Revista de Enfermagem da UFSM. 2016;6(1):102-111.

13. Araújo HVS, Figueiredo TR, Costa CRB, Silveira MMBM, Belo RMO, Bezerra SMMS. Quality of life of patients who undergone myocardial revascularization surgery. Rev Bras Enferm. 2017;70(2):257-64.

14. Cani KC, Bonorino KC, Gulart AA, Palú M, Karloh M, Mayer AF. Complicações pulmonares após cirurgia de revascularização do miocárdio: fatores associados. ASSOBRAFIR Ciência. 2017;8(2):41-50.

15. Costa VEA, Ferolla SM, Reis TOD, Rabello RR, Rocha EAV, Couto CMF, et al. Impact of body mass index on outcome in patients undergoing coronary artery bypass grafting and/or valve replacement surgery. Rev Bras Cir Cardiovasc. 2015;30(3):335-342.

16. Dessote CAM, Rodrigues HF, Furuva RK, Rossi LA, Dantas RAS. Stressors perceived by patients in the immediate postoperative of cardiac surgery. Revista Brasileira de Enfermagem. 2016;69(4):694-713.

17. Simão AF, Précoma DB, Andrade JP, Correa Filho H, Saraiva JFK, Oliveira GMM, et al. Sociedade Brasileira de Cardiologia. I Diretriz Brasileira de Prevenção Cardiovascular. Arquivos Brasileiros de Cardiologia. 2013;101(6Supl2):1-63.

18. Caldeira C, Soares AJC. Perfil clínico e epidemiológico dos pacientes que realizaram cirurgia cardíaca no hospital sul fluminense-HUSF. Revista de Saúde. 2017;8(1):03-07.

19. Kaufman R, Kuschnir MCC, Xavier RMA, Santos MA, Chaves RBM, Müller RE, et al. Perfil epidemiológico na cirurgia de revascularização miocárdica. Revista Brasileira de Cardiologia. 2011;24(6):369-76.

20. Dallazen F, Windmöller P, Winkelmann ER, Berlezi EM. Aspectos clínico-demográficos de pacientes submetidos à cirurgia cardíaca eletiva. Revista de enfermagem UFPE online. 2016;10(6):1971-1979.

21. Cordeiro ALL, Lima ASL, Matos ICO, Oliveira LVB, Guimarães AR, Carvalho SO, et al. Análise do tempo de ventilação mecânica e internamento em pacientes submetidos a cirurgia cardíaca. ABCS Health Sciences. 2017; 42(1):3-7 
22. Rocha GMM, Filho ENB. Correlação entre o uso de circulação extracorpórea, tempo de internação e complicações pulmonares pós-revascularização do miocárdio. Revista Fisioterapia e Saúde Funcional. 2012;1(2).

23. Lomivorotov VV, Efremov SM, Pokushalov EA, Karaskov AM. New-onset atrial fibrillation after cardiac surgery: pathophysiology, prophylaxis, and treatment. Journal of Cardiothoracic and Vascular Anesthesia. 2016;30(1):20016.

24. Hawkins RB, Mehaffey JH, Guo A, Charles EJ, Speir AM, Rich JB, et al. Postoperative atrial fibrillation is associated with increased morbidity and resource utilization after left ventricular assist device placement. The Journal of Thoracic and Cardiovascular Surgery. 2018;156(4):1543-1549.

25. Silva RG, Lima GG, Laranjeira ACAR, Costa ARD, Pereira E., Rodrigues R. Risk factors, morbidity, and mortality associated with atrial fibrillation in the postoperative period of cardiac surgery. Arquivos Brasileiros de Cardiologia. 2004;83(2):99-104.

26. Cavenaghi ES, Ferreira LL, Marino LHC, Lamari NM. Fisioterapia respiratória no pré e pós-operatório de cirurgia de revascularização do miocárdio. Revista Brasileira de Cirurgia Cardiovascular. 2011; 26 (3): 455-461.

27. Cavalcante ES, Magario R, Conforti CA, Junior GC, Arena R, Carvalho ACC, et al. Impact of intensive physiotherapy on cognitive function after coronary artery bypass graft surgery. Arquivos Brasileiros de Cardiologia. 2014; 103 (5): 391-397.

28. Oliveira DS, Silva RCO, Bassi D, Calles ACN. Associação entre as complicações pulmonares e fatores predisponentes em cirurgias cardiopediátricas. ConScientiae Saúde. 2017;16(4):441-446.

29. Brown KL, Ridout D, Pagel C, Wray J, Anderson D, Barron DJ, et al. Incidence and risk factors for important early morbidities associated with pediatric cardiac surgery in a UK population. Thorac Cardiovasc Surg. 2019 Jun $12 .$. [Epub ahead of print]. 